\title{
Nitrosative Stress Is Associated with Dopaminergic Dysfunction in the HIV-1 Transgenic Rat
}

Swati Shah, ${ }^{*}$ Dragan Maric, ${ }^{\dagger}$ Frank Denaro, ${ }^{\ddagger}$ Wael Ibrahim, ${ }^{*}$ Ronald Mason, ${ }^{\S}$ Ashutosh Kumar, ${ }^{\S}$ Dima A. Hammoud, ${ }^{*}$ and William Reid*

From the Center for Infectious Disease Imaging, Radiology and Imaging Sciences, * Clinical Center, and the National Institute of Neurological Disorders and Stroke, ${ }^{\dagger}$ NIH, Bethesda, Maryland; the Department of Biology, ${ }^{\ddagger}$ Morgan State University, Baltimore, Maryland; and the Free Radical Biology Group, Immunity, Inflammation, and Disease Laboratory, ${ }^{\S}$ National Institute of Environmental Health Sciences, NIH, Durham, North Carolina

\author{
Accepted for publication \\ March 7, 2019. \\ Address correspondence to \\ Dima A. Hammoud, M.D., \\ NIH, 10 Center Dr., Bethesda, \\ MD 20892. E-mail: \\ hammoudd@cc.nih.gov.
}

\begin{abstract}
Advances in antiretroviral therapy have resulted in significantly decreased HIV-related mortality. HIVassociated neurocognitive disorders, however, continue to be a major problem in infected patients. The neuropathology underlying HIV-associated neurocognitive disorders has not been well characterized, and evidence suggests different contributing mechanisms. One potential mechanism is the induction of oxidative stress. Using the HIV-1 transgenic $(\mathrm{Tg})$ rat model of HIV, we found increased striatal NADPH oxidase- 4 and neuronal nitric oxide synthase expression in the adult (7- to 9-month-old) $\mathrm{Tg}$ rat compared with control rats but not in the young (1-month-old) $\mathrm{Tg}$ rats. This was accompanied by increased 3-nitrotyrosine (3-NT) immunostaining in the adult $\mathrm{Tg}$ rats, which worsened significantly in the old $\mathrm{Tg}$ rats (18 to 20 months old). There was, however, no concurrent induction of the antioxidant systems because there was no change in the expression of the nuclear factor-erythroid 2-related factor 2 and its downstream targets (thioredoxin and glutathione antioxidant systems). Colocalization of 3-NT staining with neurofilament proteins and evidence of decreased tyrosine hydroxylase and dopamine transporter expression in the old rats support dopaminergic involvement. We conclude that the HIV-1 Tg rat brain shows evidence of nitrosative stress without appropriate oxidation-reduction adaptation, whereas 3-NT modification of striatal neurofilament proteins likely points to the ensuing dopaminergic neuronal loss and dysfunction in the aging HIV-1 Tg rat. (Am J Pathol 2019, 189: 1375 -1385; https://doi.org/10.1016/j.ajpath.2019.03.004)
\end{abstract}

Although the introduction of highly active antiretroviral therapy has significantly decreased the incidence of HIVassociated dementia complex, an incapacitating consequence of untreated HIV infection, ${ }^{1-3}$ less severe forms of HIVassociated neurologic disorders have become more common and currently affect approximately $30 \%$ to $50 \%$ of the HIVpositive patients. ${ }^{3,4}$ The suspected mechanisms underlying HIV-associated neurologic disorders include persistent neuroinflammation, ${ }^{5}$ neurotoxic effects of HIV-1 viral proteins, ${ }^{6-8}$ disruption of the neurotrophic function of astrocytes and oligodendrocytes,,${ }^{9,10}$ as well as the production of reactive oxygen species (ROS) and reactive nitrogen species (RNS) and the underproduction of antioxidants. ${ }^{11-17}$ Related to the latter mechanism, reports indicate that HIV infection hastens the normal aging-related neurodegenerative process by accelerating neuronal excitotoxicity, atypical $\mathrm{Ca}^{+}$ signaling, and dysregulated production of ROS/RNS that contribute to oxidative/nitrative stress (OS/NS). ${ }^{18-20}$ This imbalance in the oxidation-reduction (redox) state can be cytotoxic to neurons through the inhibition of cellular respiration, dysregulation of mitochondrial electron transport, and

\footnotetext{
Supported by the Center for Infectious Disease Imaging, the Clinical Center Intramural Research Program, and the National Institute of Allergy and Infectious Diseases at the NIH grant ZIA CL090037-02.

D.A.H. and W.R. contributed equally to this work as senior authors.

Disclosures: None declared.

Current address of W.I., Division of Medical Oncology, University of Kentucky Healthcare Markey Cancer Center, Lexington, KY.
} 
posttranslational modification of neuronal structural proteins. $^{21-24}$

Normally, a basal level of ROS/RNS is required to maintain cellular functions. ${ }^{25}$ An overproduction of these free radicals, however, can cause damage to proteins, lipids, and DNA. ${ }^{26}$ Superoxide, hydrogen peroxide, hydroxyl radicals, nitric oxide, and peroxynitrite ${ }^{27,28}$ are major oxidants implicated in various neurodegenerative disorders. Major sources of ROS that contribute to the pathology of acute brain injury and chronic neurodegenerative disorders are various NADPH oxidases (NOXs). ${ }^{29,30}$ NOX proteins include seven members (NOX1-NOX5 and dual oxidase 1 and 2) that are membrane-bound enzyme complexes playing a critical role in oxidative bursts. ${ }^{27,28}$ NOX4 is unique among the NOX isoforms as it constitutively produces measurable amounts of hydrogen peroxide. ${ }^{30}$ NOX4 is also ubiquitously expressed in a variety of tissues and has been shown to mediate signal transduction pathways by modulating intracellular $\mathrm{Ca}^{+}$levels. ${ }^{31}$ Depletion of Nox4 expression was found to reduce oxidative damage to nuclear DNA and delay senescence in endothelial cells. ${ }^{32,33}$

A major source of RNS is peroxynitrite, produced by the reaction of nitric oxide and superoxide. Peroxynitrite is a destructive oxidant and nitrating agent, capable of oxidizing lipoproteins and causing the nitration of selective tyrosine residues in proteins, thereby altering protein structure, conformation, and function. ${ }^{34,35} 3$-Nitrotyrosine (3-NT) formation above basal levels indicates nitrosative stress, which correlates with disease progression and severity. On the basis of buffering conditions in the biological milieu, peroxynitrite decomposes and forms highly active free radicals, such as hydroxyl radicals, carbon trioxide, and nitrogen dioxide. These highly reactive products are more efficient nitrating species than peroxynitrite itself. ${ }^{21}$ In fact, nitrogen dioxide is a major contributor to the formation of 3-NT modified proteins. ${ }^{36}$ Nitric oxide is synthesized by the nitric oxide synthase (NOS) enzyme that exists as three isoforms: neuronal NOS (nNOS), inducible NOS, and endothelial NOS. Neuronal NOS is a major source of NOS in the brain and is regulated transcriptionally and translationally. ${ }^{21,37,38}$

Under normal conditions, ROS/RNS and subsequent OS/NS are kept under control through the antioxidant system. ${ }^{39}$ However, under disease conditions, cells are prone to OS/NS damage, likely as a result of inadequate redox adaptation. ${ }^{40,41}$ Nuclear factor-erythroid 2-related factor 2 (Nrf2) is a transcription factor that is induced by OS/NS and binds to the antioxidant response elements of phase 2 antioxidant enzymes. Nrf2 positively regulates the expression of antioxidants that protect cells from oxidative stress. ${ }^{39,42}$ Both the glutathione and thioredoxin (TRX) enzyme systems are phase 2 antioxidants and protect cells from damage caused by ROS/RNS. Glutathione biosynthesis is regulated by Nrf2 in both neurons and astrocytes. $^{39,43}$ The glutathione antioxidant system is responsible for scavenging multiple nitrosative species and reducing lipid hydroperoxides. ${ }^{39,44}$ The TRX system is directly involved in reducing several substrates, including lipid hydroperoxides and hydrogen peroxide. ${ }^{45-48}$ Curiously, not only does Nrf2 positively regulate expression of phase 2 antioxidants but it also is a positive regulator of enzymes regulating NADPH, $\mathrm{NADH}, \mathrm{FADH}_{2}$, mitochondrial membrane potential, and respiration. ${ }^{22}$ Therefore, a dysregulation in Nrf2 activity likely alters both cellular respiration and redox homeostasis.

The HIV-1 transgenic (Tg) rat is a well-characterized small animal model of HIV that has been proposed as a model for treated HIV-positive patients. ${ }^{16,49-52}$ This noninfectious rat model expresses seven of the nine HIV viral proteins (including gp120 and Tat), has been reported to develop neuropathology and cognitive deficits, ${ }^{52-56}$ and demonstrates regional variations in redox adaptation, mitochondrial dysfunction, and neurotoxicity. ${ }^{16,57-60}$ Our previous research using this animal model, which includes imaging as well as behavioral and histologic assessments, supports dopaminergic dysfunction in the $\mathrm{Tg}$ rat. $^{54,61-63}$ The goal of the current study is to investigate a possible link between this dopaminergic dysfunction and OS/NS. Therefore, striatal NOX4 and nNOS expression was measured, and the extent of nitration of tyrosine residues in neurofilament proteins of the Tg rats compared with age-matched controls was evaluated. The dopaminergic system of old Tg and control rats was also evaluated using immunohistochemistry by looking at tyrosine hydroxylase (TH), dopamine transporter (DAT) in the striatum, and NeuN (a neuronal marker) in the substantia nigra. Finally, the induction of Nrf2 and members of the phase 2 glutathione and TRX antioxidant pathways was evaluated to assess the integrity of the expected secondary antioxidant response.

\section{Materials and Methods}

\section{Animals}

Male HIV-1 Tg (F344/Hsd, Tg) and age-matched wild-type control (F344/Hsd) rats were purchased from Envigo Inc. (Indianapolis, IN). All rats were housed in a temperaturecontrolled environment with a 12-hour light/dark cycle and free access to food and water. All studies were approved by the Animal Care and Use Committee of the NIH/Clinical Center (Bethesda, MD). Three animal cohorts of different ages were used for various experiments: 1-month-old (young), 7- to 9month-old (adult), and 18- to 20-month-old (old) rats.

\section{0xidative Stress $\mathrm{RT}^{2}$ Profiler PCR Array Assay}

Total cellular RNA from young ( $n=7$ each) and adult $(n=6$ each) $\mathrm{Tg}$ animals and controls was extracted from striatal tissue using an RNeasy Lipid Tissue minikit (Qiagen, Valencia, CA), according to the manufacturer's instructions. Synthesis of cDNA was performed using $\mathrm{RT}^{2}$ First Strand Kit and the cDNA was then used on the Rat Oxidative Stress Real-Time $\mathrm{RT}^{2}$ Profiler PCR Array (Qiagen; catalog number PARN-065Z) in combination with $\mathrm{RT}^{2}$ SYBR Green qPCR Mastermix (Qiagen). Relative changes in expression levels of 
specific mRNA were quantified using the CFX96 Real-Time qPCR System (Bio-Rad, Hercules, CA). $\mathrm{C}_{\mathrm{t}}$ values were normalized to a selection of housekeeping gene panels, and fold changes were calculated using the $\Delta \Delta \mathrm{C}_{\mathrm{t}}$ method. The $\mathrm{RT}^{2}$ RNA Quality Control PCR Array was performed on each sample before $\mathrm{RT}^{2}$ Profiler PCR Array Assay, according to the manufacturer's instructions (PARN-999Z). Each gene identified with at least a 1.5 -fold change in expression by the PCR Array was further validated using individual real-time quantitative PCR (qPCR) assays. The qPCR primer sets used for further gene validation were as follows: Nox4 (PPR45975A), hemoglobin $\alpha$ adult chain 2 (Hbal; PPR56540A), uncoupling protein 3 (Ucp3; PPR44548A), and albumin (Alb; PPR48689A). Separately, an individual qPCR assay was also performed for Nrf2 (PPR45094A).

\section{Western Blot Analysis}

Aliquots $(20 \mu \mathrm{g})$ of striatal lysates from young (control, $n=3$; $\mathrm{Tg}, n=4$ ) and adult (control, $n=3$; $\mathrm{Tg}, n=4$ ) animals were run on Any KD TGX SDS-PAGE gels (Bio-Rad) and transferred to polyvinylidene difluoride membranes, as previously described. ${ }^{64}$ The primary antibodies used were NOX4 (Abcam, Cambridge, UK; catalog number ab133303; dilution 1:3000), nNOS (Abcam; catalog number ab95436; $1 \mu \mathrm{g} / \mathrm{mL}$ ), Nrf2 (Abcam; catalog number ab89443; $1 \mu \mathrm{g} / \mathrm{mL}$ ), and actin (CST, Danvers, MA; catalog number 8457S; dilution 1:1000). The secondary antibodies used were goat anti-rabbit (Jackson Laboratory, West Grove, PA; catalog number 111-035-003; dilution 1:50,000) and goat anti-mouse (Jackson Laboratory; catalog number 115-035-174; dilution 1:50,000). All antibodies, except actin, were diluted in 5\% milk with Tris-buffered saline containing Tween 20 . The actin antibody was diluted in $5 \%$ bovine serum albumin with Tris-buffered saline containing Tween 20.

\section{Immunofluorescence}

HIV-1 Tg brain sections were evaluated using immunofluorescence and compared with age-matched control rat brains in the adult (control, $n=4 ; \mathrm{Tg}, n=6$ ) and old (control, $n=5$; $\mathrm{Tg}, n=4)$ animal cohorts, as previously described. ${ }^{61}$ The animals were anesthetized with isoflurane (3\% with $700 \mathrm{~mL} /$ minute $\mathrm{O}_{2}$ ), followed by transcardial perfusion with saline and $4 \%$ paraformaldehyde. The brains were removed, and the striatal and substantia nigra sections (bregma, 0.48 to $0.12 \mathrm{~mm}$ and -2.92 to $-3.24 \mathrm{~mm}$, respectively) were then selected for immunofluorescence staining, as per the atlas of Paxinos and Watson. ${ }^{65}$ Staining protocols were used to identify microglia using ionized calcium binding adaptoer molecule 1 (IBA-1; Wako Chemicals, Richmond, VA; catalog number 01919741), tyrosine hydroxylase (Novus Biologicals, Centennial, CO; catalog number NB300-110), dopamine transporter (DAT extracellular; Santa Cruz Biotechnology, Dallas, TX; catalog number sc-32259), neuronal soma (NeuN; EMD Millipore, Burlington, $\mathrm{MA}$; catalog number ABN90P), reactive astrocytes [glial fibrillary acidic protein (GFAP); US Biological, Salem, MA; catalog number G2032-27Q], and nitrative stress (3-nitrotyrosine; Abcam; catalog number ab110282). Multiepitope labeling protocols were then applied to identify the above targets and imaged, as previously described. ${ }^{63}$ After postprocessing, the data sets were imported into Adobe Photoshop CS6 (Adobe Inc., San Jose, CA) to produce pseudocolored composite images.

Image quantification was then performed using the FIJI implementation of ImageJ version 1.6.0 (NIH; https:// imagej.nih.gov/ij). The locations of the striatal and substantia nigra regions of interest were identical for all of the animals, and the fluorescence intensity was calculated as described previously. ${ }^{63}$ The data are expressed as percentage fluorescence intensity relative to the area of the region of interest for all stains.

\section{Confocal Microscopy}

Frozen striatal brain sections from old male $\operatorname{Tg}(n=3)$ and control $(n=3)$ rats $(30 \mu \mathrm{m}$ thick) were evaluated using immunofluorescence labeling for colocalization of 3-NT to a neurofilament cocktail consisting of light, medium, and heavy chains, using Zeiss LSM 780 with a $63 \times$ Zeiss planapochromat oil, 1.4 numerical aperture, differential interference contrast objective (Zeiss, Jena, Germany). Striatal sections were stained with cocktails of neurofilament antibodies (Abcam; catalog numbers ab9035, ab9034, and ab8135) and anti-3-NT (Abcam; catalog numbers ab61392 and ab1 10282). The neurofilaments and 3-NT were visualized using goat antirabbit IgG Alexa Fluro 488 (Invitrogen, Carlsbad, CA; catalog number A11008) and goat anti-mouse IgG2b-Alexa Fluor 647 (Invitrogen; catalog number A21242) secondary antibodies, respectively. The cell nuclei were counterstained using $1 \mu \mathrm{g} / \mathrm{mL}$ DAPI. All confocal images were acquired using ZEN software 2011 version (Zeiss), in which 20 nonoverlapping fields per section (two sections per slide) were analyzed for colocalization; these data were converted to percentage colocalization per animal for statistical analysis.

\section{Statistical Analysis}

Statistical significance was determined by unpaired twotailed $t$-test or two-way analysis of variance with Sidak's multiple comparisons test using Prism GraphPad version 7 (GraphPad Software, San Diego, CA). All data are represented as means \pm SEM. Correlations and simple linear regression analysis were determined by Pearson correlation coefficient. $P<0.05$ was considered significant.

\section{Results}

Dysregulated Expression of Oxidative Stress Markers in Adult Tg Rats

Our previous data supported dopaminergic dysfunction as the Tg rat ages. ${ }^{54,61-63}$ We hypothesized that chronic oxidative 
stress in the striatum is one of the causes for those findings. Using a PCR array, only 4 of 84 genes were found to be differentially expressed above or below the 1.5 -fold cutoff: Nox4, Hbal, Ucp3, and Alb. Specifically, Nox4 expression was found to be increased by 1.6- and 2-fold in the young and adult $\mathrm{Tg}$ rats, respectively (Table 1 ). Further evaluation using a specific qPCR assay for Nox4 showed slightly increased gene transcription in the young $\mathrm{Tg}$ compared with agematched control rats, which became more marked (approximately 2-fold) in the adult $\mathrm{Tg}$ compared with age-matched control rats (Figure 1A). However, although those differences were not statistically significant, the differences in the adult group approached significance $(P=0.06)$. On the other hand, no significant differences in the expression of Hbal, $U c p 3$, and $A l b$ were identified (data not shown).

To further follow up on the dysregulation in Nox 4 mRNA levels, protein expression of these genes was quantified using Western blot analysis in the same animals. NOX4 protein expression in the young cohort was not significantly different from the control rats (Figure 1C). A significant elevation in NOX4 protein expression, however, was detected in the adult Tg rats $(P=0.02)$ compared with agematched control rats (Figure 1C). Using a two-way analysis of variance analysis of NOX4 expression in the young and adult cohorts, a significant age $\times$ transgene effect interaction was found $[F(1,10)=5.6, P=0.04]$.

Table 1 Striatal 0xidative Stress Response: $\mathrm{RT}^{2}$ Profiler PCR Array

\begin{tabular}{lll}
\hline & \multicolumn{2}{l}{$\begin{array}{l}\text { Fold change for transgenic } \\
\text { versus control }\end{array}$} \\
\cline { 2 - 3 } Gene symbol & Young & Adult \\
\hline Nox4 & 1.6 & 2.0 \\
Alb & 3.0 & 1.5 \\
Gclc & 1.2 & 1.4 \\
Gclm & 1.1 & 1.2 \\
Gpx1 & 1.1 & 1.2 \\
Gpx2 & 1.2 & 1.4 \\
Gpx3 & 1.1 & 1.3 \\
Gpx4 & 1.1 & 1.3 \\
Gpx7 & 1.1 & 1.1 \\
Gsr & 1.1 & 1.3 \\
Gstk1 & 1.0 & 1.1 \\
Gstp1 & 1.0 & -1.1 \\
Hba1 & -2.3 & 7.8 \\
Prdx1 & 1.0 & 1.1 \\
Prdx2 & -1.0 & 1.2 \\
Prdx3 & -1.0 & 1.2 \\
Prdx4 & 1.0 & 1.1 \\
Prdx5 & 1.1 & 1.3 \\
Prdx6 & 1.0 & 1.3 \\
Srxn1 & -1.1 & 1.2 \\
Txn1 & 1.1 & 1.1 \\
Txnrd1 & 1.1 & 1.3 \\
Txnrd2 & -1.1 & 1.2 \\
Ucp3 & -1.3 & 2.9 \\
\hline
\end{tabular}

Dysregulated Antioxidant Response in the Adult Tg Rats

In view of the above findings, $N r f 2$ expression was assessed in the adult animals using qPCR as a measure of the antioxidant response because Nrf2 is the master regulator of $G s h$ and $\operatorname{Tr} x$ antioxidant gene transcription, and its expression is expected to increase in response to NOX4 up-regulation. Unexpectedly, the relative change in Nrf2 mRNA expression in the adult $\mathrm{Tg}$ and control rats was not significantly different (Figure 1B). Consistent with Nrf2 mRNA expression, no significant difference in Nrf2 protein levels was detected between adult Tg and control rats (Figure 1D). Similarly, no increase in the expression of Gsh and Trx antioxidants was seen in the Tg rats by the PCR array (Table 1).

\section{Nitrative Stress in the Basal Ganglia of Tg Rats Increases with Age}

To assess for NS in the striatum in the young and adult Tg rats, levels of nNOS protein were measured by Western blot analysis. In the young cohort, no significant difference was identified between $\mathrm{Tg}$ and age-matched control rats; in the adult $\mathrm{Tg}$ rats, on the other hand, a significant 3.4-fold increase in striatal nNOS protein expression was identified relative to agematched controls $(P=0.0005)$ (Figure 2$)$. A two-way analysis of variance analysis showed a highly significant age $\times$ transgene effect interaction $[F(1,10)=18 ; P=0.002]$.

\section{Immunohistochemical Evidence of Dopaminergic Dysfunction}

Immunohistochemical analysis of striatal brain tissues showed increased 3-NT staining in adult and old Tg rats compared with their corresponding age-matched controls. This was highly significant in the old $\mathrm{Tg}$ compared with control rats $(P=0.0003)$ but not significant in the adult cohort (Figure 3A). Again, a two-way analysis of variance analysis found a significant age $\times$ transgene effect interaction $[F(1,15)=8.2$; $P=0.01]$. This suggests that the normal age-related buildup of nitration is exacerbated in the $\mathrm{Tg}$ rats. In view of the pattern of 3NT staining, which seemed to follow the axonal tracts, we hypothesized that neurofilament proteins would be a likely target for nitration. Using confocal microscopy, an approximately $40 \%$ increase in tyrosine nitration of striatal neurofilament proteins $(P=0.01)$ was identified in old $\mathrm{Tg}$ rats relative to controls (Figure 3B). These data suggest that the dysregulation of neuronal function in the aging $\mathrm{Tg}$ rat might be related to NS and that the posttranslational modification of neurofilament proteins could subsequently lead to neuronal loss.

\section{Presynaptic Dopaminergic Dysfunction and Astrocytic Loss in the Tg Rats}

To further assess potential effects of NS on the presynaptic dopaminergic neurons in the old animals, multiplex immunohistochemical analysis of various targets in the brains of old 
A

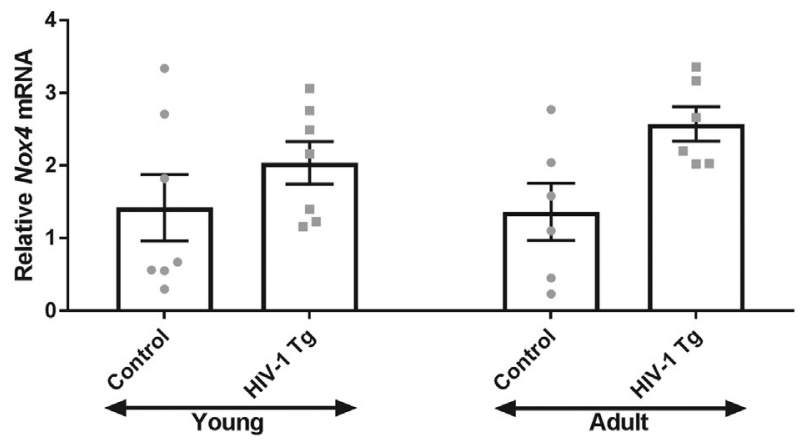

\section{C}
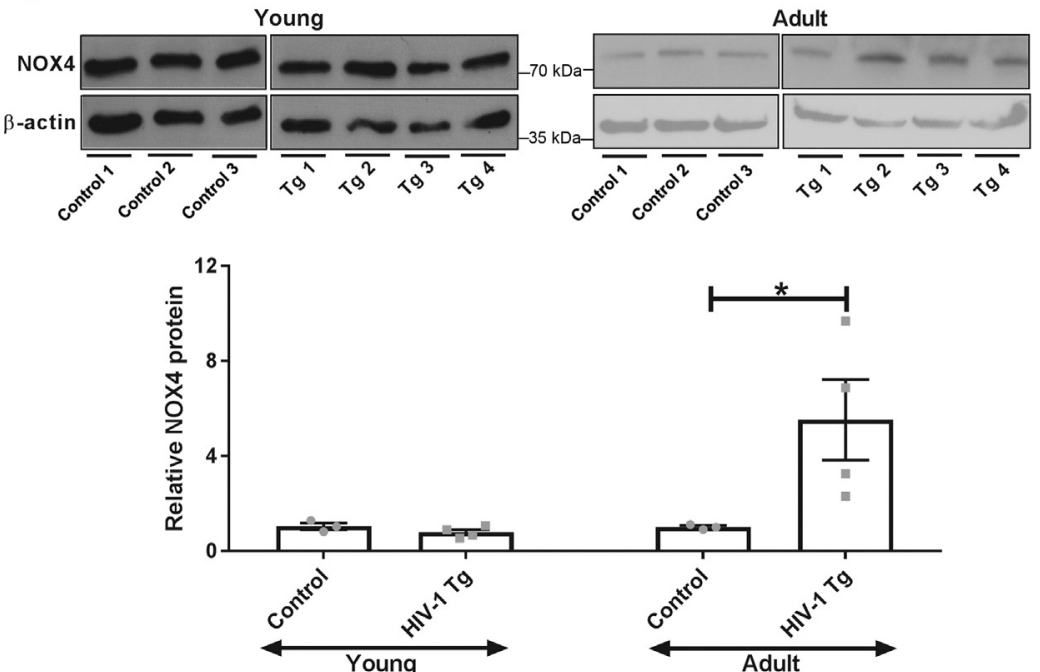

B

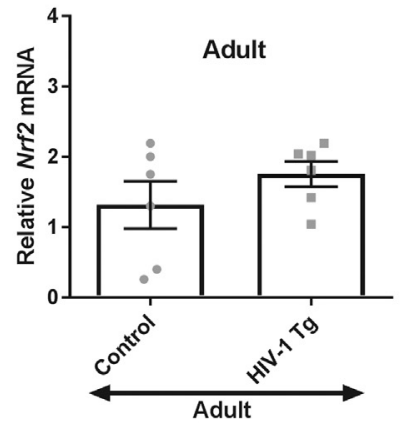

D
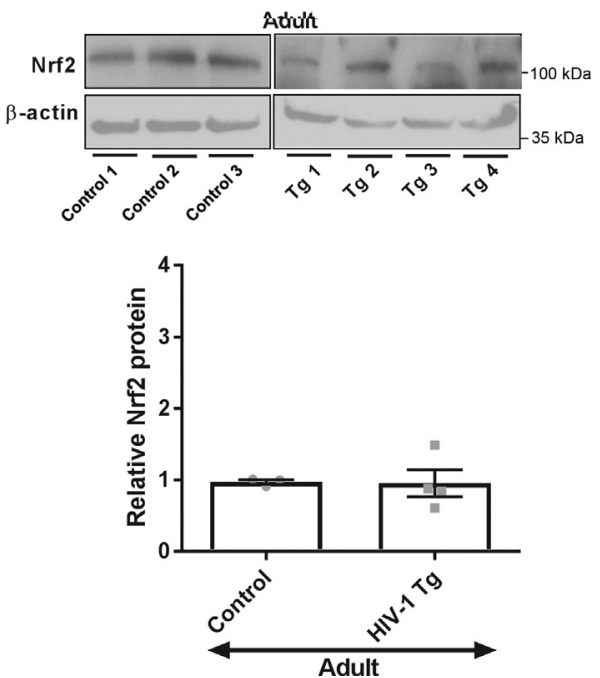

Figure 1 Characterization of striatal oxidative stress response in HIV-1 transgenic (Tg) rats. Real-time quantitative PCR assessment of Nox4 mRNA expression in young and adult HIV-1 Tg rats relative to controls (A) and nuclear factor-erythroid 2-related factor 2 (Nrf2) mRNA expression in adult HIV-1 Tg rats relative to control (B). Western blot analysis for the determination of protein expression of NADPH oxidase-4 (NOX4) in young and adult HIV-1 Tg rats relative to controls (C) and Nrf2 in adult HIV-1 Tg rats relative to controls (D). Western blot analyses were quantified using FIJI (ImageJ version 1.6.0) and are shown below the respective blots. Statistical analysis of NOX4 expression in young and adult cohorts was performed using two-way analysis of variance with Sidak multiple comparisons test. Nrf2 expression data were analyzed by $t$-test. $* P<0.05$.

control and Tg rats was performed. As expected, a significantly lower expression of DAT $(P=0.02)$, TH $(P=0.0003)$, and GFAP $(P=0.03)$ was identified in old Tg rats, indicating dopaminergic and astrocytic dysfunction (Figure 4, A-C). A significant negative correlation was observed between the percentage fluorescence intensities of striatal 3-NT versus DAT, TH, and GFAP fluorescence intensities (Figure 4, D-F).

In addition to the striatal defects, a significant increase in 3-NT staining $(P=0.03)$ and a concomitant decrease in NeuN-positive cells $(P=0.03)$ were also identified in the substantia nigra of the old $\mathrm{Tg}$ rats relative to age-matched controls (Figure 5, A and B), indicating neuronal loss. However, no significant correlation was observed between 3-NT and NeuN staining (Figure 5C).

\section{Discussion}

There has always been an interest in the role of oxidative/ nitrative stress in the pathophysiology of HIV-associated dementia complex and HIV-associated neurologic disorders, ${ }^{15,17,66}$ its association with viral protein expression, ${ }^{67-79}$ and how it affects the dopaminergic system. ${ }^{7,67,80}$ Herein, the HIV-1 Tg rat, a known model of viral protein toxicity, was used to assess the relationship between OS/NS markers and the end result of dopaminergic dysfunction and damage. We were mainly motivated by our previous findings in the HIV- $1 \mathrm{Tg}$ rat of decreased brain volumes, lower striatal TH, GFAP, and DAT expression, decreased striatal DAT and dopaminergic receptor (D2/D3) binding on positron emission tomography, and motor and behavioral deficits consistent with dysregulation in dopaminergic function. ${ }^{61-63,81,82}$ Those markers of dopaminergic loss/dysfunction were generally exacerbated with age in this animal model, with progressive loss of DAT, $\mathrm{TH}$, and D2/D3 receptors in the basal ganglia of $\mathrm{Tg}$ compared with control rats shown on either positron emission tomography imaging or immunohistochemistry. ${ }^{54,61,63}$ As expected, evidence of OS/NS was found in this study with increased 3-NT staining in 

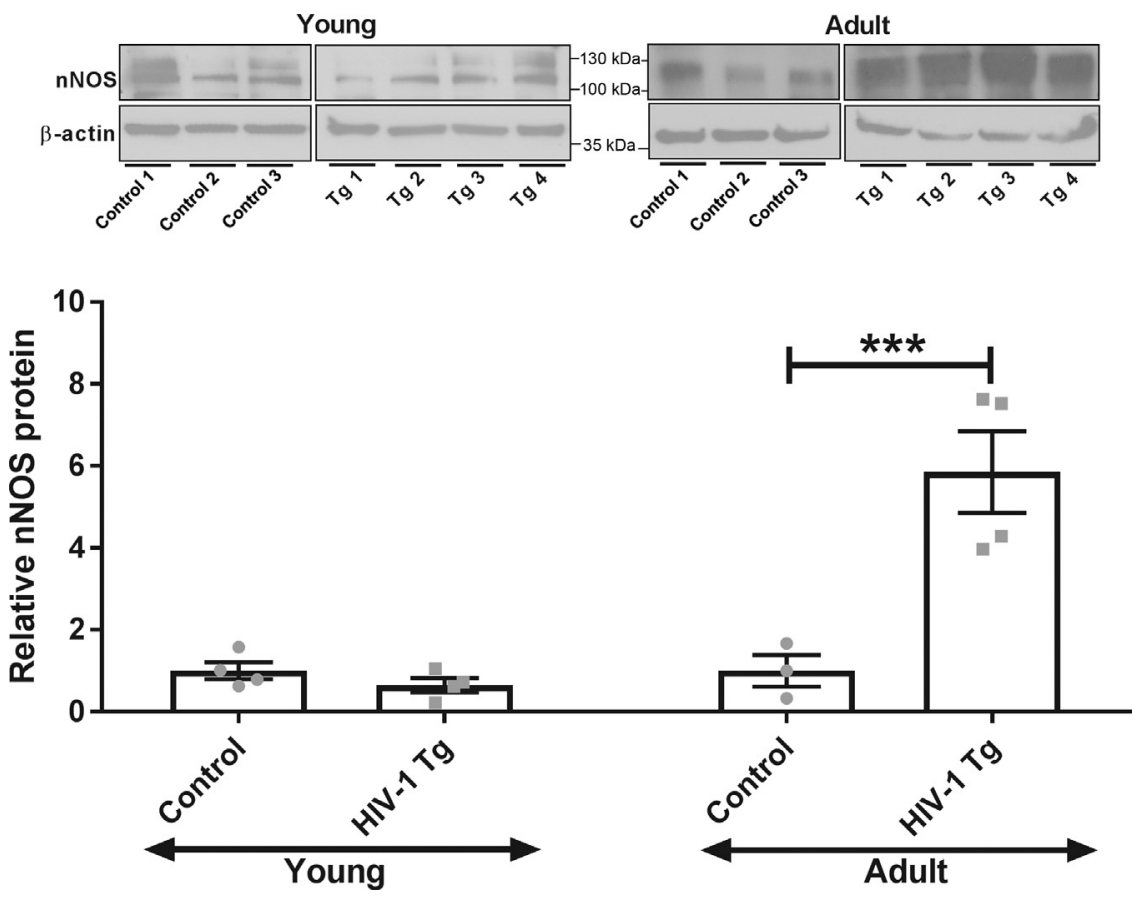

Figure 2 Characterization of neuronal nitric oxide synthase (nNOS) response in the HIV-1 transgenic $(\mathrm{Tg})$ rats. nNOS protein expression in HIV-1 Tg rats relative to controls, as shown by Western blot analysis with quantification in young and adult rats. Quantification data were analyzed using two-way analysis of variance with Sidak multiple comparisons test. ${ }^{* *} P<0.001$.

the striatum of adult and old $\mathrm{Tg}$ rats associated with aging and transgene status (Figure 3A). Concurrently, decreased presynaptic $\mathrm{TH}$ and DAT staining was found in the striatum of old $\mathrm{Tg}$ rats with significant negative correlation between the fluorescence intensities of 3-NT and $\mathrm{TH}$ (Figure 4, A and D) and 3-NT and DAT (Figure 4, B and E). At the same time, a decrease in striatal GFAP staining, which also negatively correlated
A

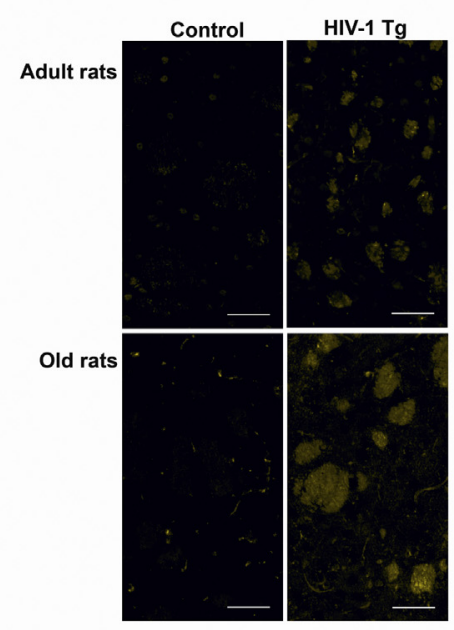

B
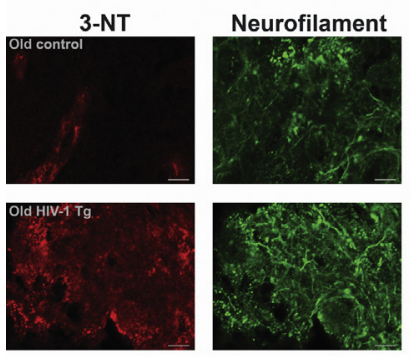
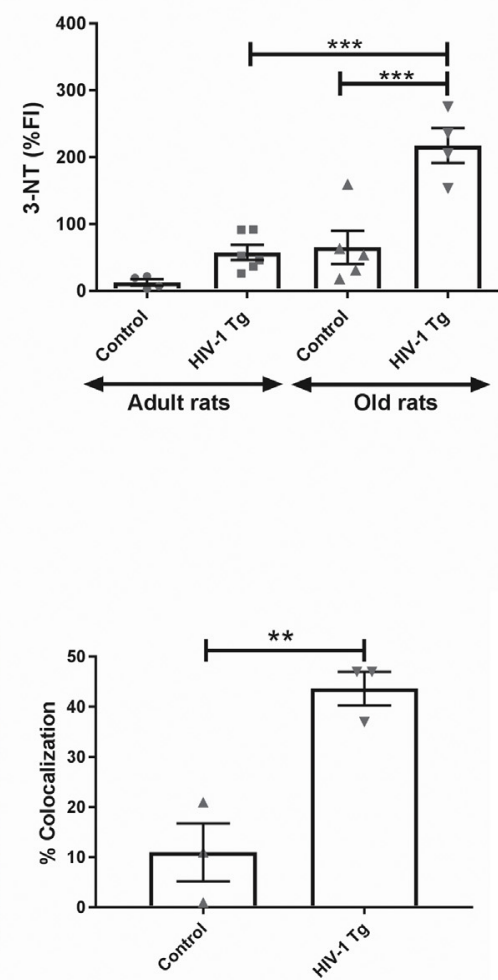

Figure 3 Characterization of nitrative stress response in the HIV-1 transgenic (Tg) rats. A: 3Nitrotyrosine (3-NT) staining of striatal tissue sections from adult and old animal cohorts. Quantification data are represented as percentage fluorescence intensity (\%FI) and analyzed using two-way analysis of variance with Sidak's multiple comparisons. B: Confocal microscopy was performed to assess percentage colocalization between 3-NT and neurofilament proteins in the striatal region of old HIV-1 $\mathrm{Tg}$ and control animals. Data were analyzed using $t$-test. ${ }^{* *} P<0.01$, $* * * P<0.001$. Scale bars: $100 \mu \mathrm{m}(\mathbf{A}) ; 5 \mu \mathrm{m}$ (B). 
A
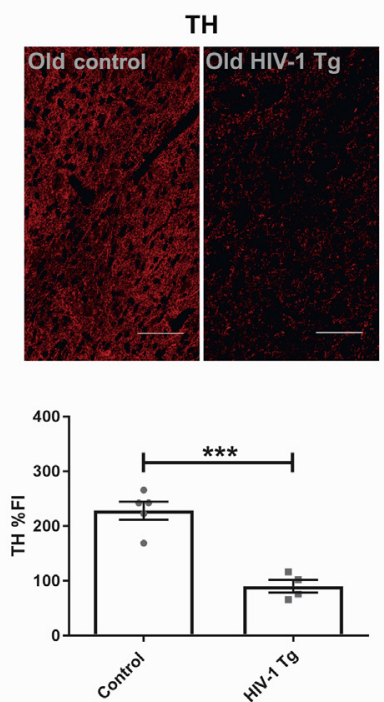

D

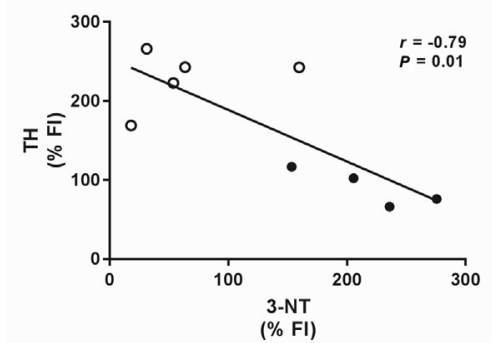

B
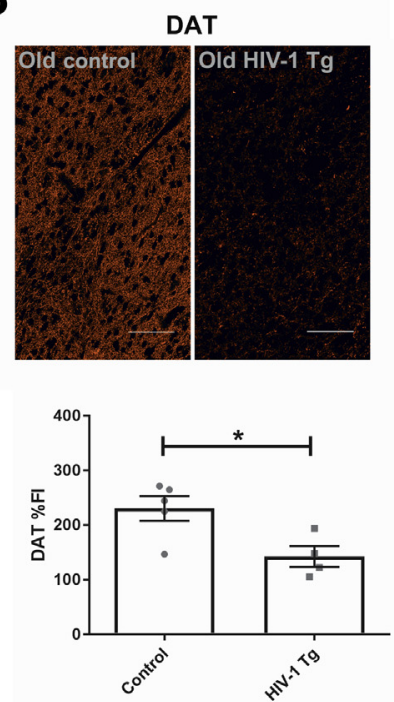

E

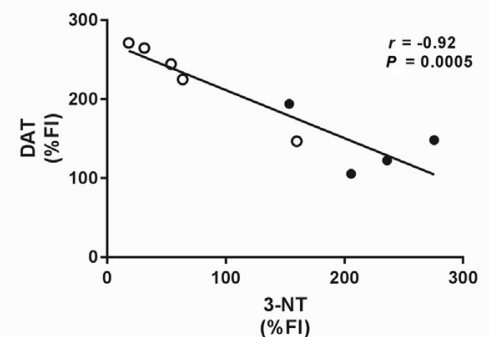

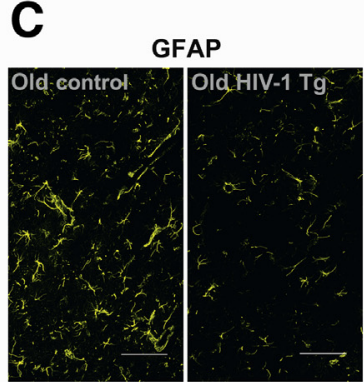

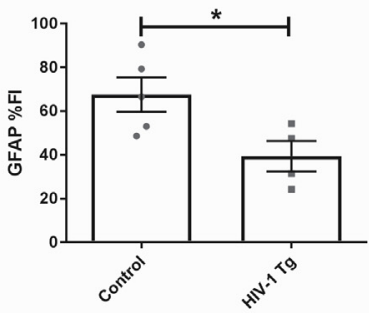

$\mathbf{F}$

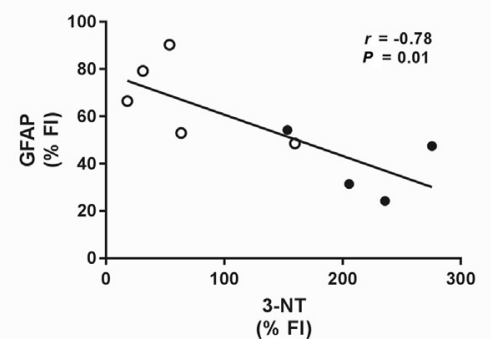

Figure 4 Cellular damage in HIV-1 transgenic ( $\mathrm{Tg}$ ) rats. Immunostaining of striatal regions of old HIV-1 Tg and control rats with tyrosine hydroxylase $(\mathrm{TH} ; \mathrm{A})$, dopamine transporter (DAT; B), and glial fibrillary acidic protein (GFAP; C).All quantification data are represented as percentage fluorescence intensity (\%FI) and analyzed using $t$-test. Correlation of 3-nitrotyrosine [3-NT (\% FI)] in striatal regions of the old rat cohort with TH (\%FI; D), DAT (\%FI; E), and GFAP $(\% \mathrm{FI} ; \mathbf{F})$. Pearson correlation coefficient $(r)$ and the $P$ values are indicated in the graphs. Open circles indicate control rats; closed circles, HIV-1 Tg rats. The $P$ values for control versus HIV-1 Tg animals are indicated. $n=9$ old rats $(\mathbf{D}-\mathbf{F}) .{ }^{*} P<0.05,{ }^{* *} P<0.001$. Scale bars $=100 \mu \mathrm{m}(\mathbf{A}-\mathbf{C})$.

with 3-NT staining, indicating concurrent astrocytic loss, was observed (Figure 4, C and F).

At the molecular level, borderline increases were found in Nox4 (mRNA) and significant increases were found in NOX4 and nNOS protein expression in the striatum of the adult but not young $\mathrm{Tg}$ rats compared with age-matched controls (Figures 1, A and C, and Figure 2). Nox4 induction can be mediated by gp120 and Tat HIV-1 proteins ${ }^{32,83}$; and the expression of gp120 and tat mRNA in striatal brain lysates as well as gp120 in the cerebrospinal fluid of the Tg rat has been shown, suggesting local viral protein production in the brain. ${ }^{62}$ Consistent with published in vitro data, these findings may reflect a pattern of OS/NS induced by chronic exposure to HIV-1 proteins in the Tg rat. Downstream effects would include NOX4-mediated production of oxidants, such as hydrogen peroxide, superoxide, and nNOS-dependent production of peroxynitrite. The latter would go on to react with other molecules and form additional types of RNS, including nitrogen dioxide and dinitrogen trioxide, causing further mitochondrial electron transport chain dysregulation, protein nitration, and neuronal injury. ${ }^{21,49,50,84}$ Alternatively, Villeneuve et al ${ }^{60}$ have demonstrated that, in the HIV-1 Tg rat, viral proteins directly enhance neuronal mitochondrial energy demand and also reduce expression of mitochondrial electron transport chain subunit proteins, likely increasing superoxide production. Some other studies have reported that increases in mitochondrial metabolism can direct the release of hydrogen peroxide and nitric oxide to the cytosol, whereas electrons prematurely exit the electron transport chain to further augment superoxide levels and associated cellular nitrosative stress. ${ }^{85-89}$ Interestingly, gp120 and Tat are also reported to increase intracellular $\mathrm{Ca}^{2+}$ levels, which can subsequently activate nNOS. ${ }^{31,90-92}$ Although Cho et $\mathrm{al}^{57}$ described the induction of inducible NOS as the molecular source of nitric oxide and nitration of heat shock protein 90 from hippocampal lysates in the HIV-1 Tg rat, the presence of inducible NOS (or endothelial NOS) in the striatal lysates of either the control or the HIV-1 Tg rats was not detected. 

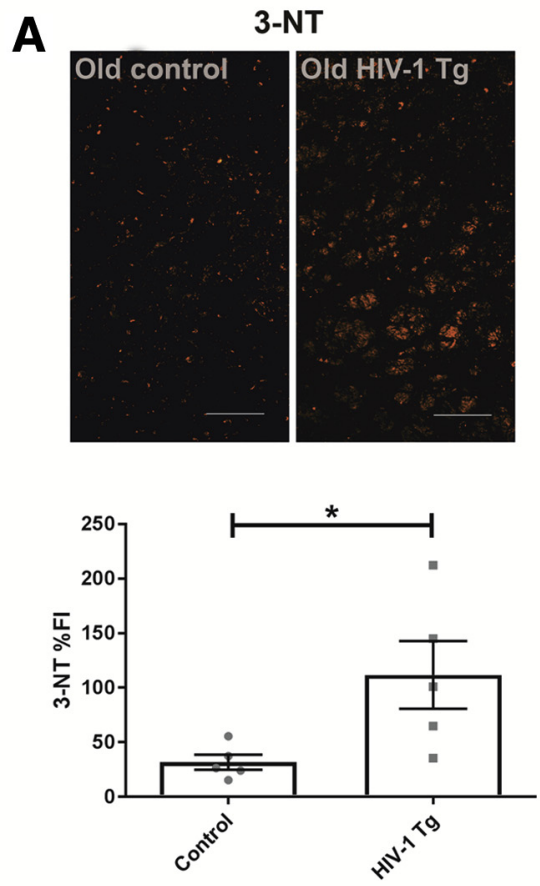

B
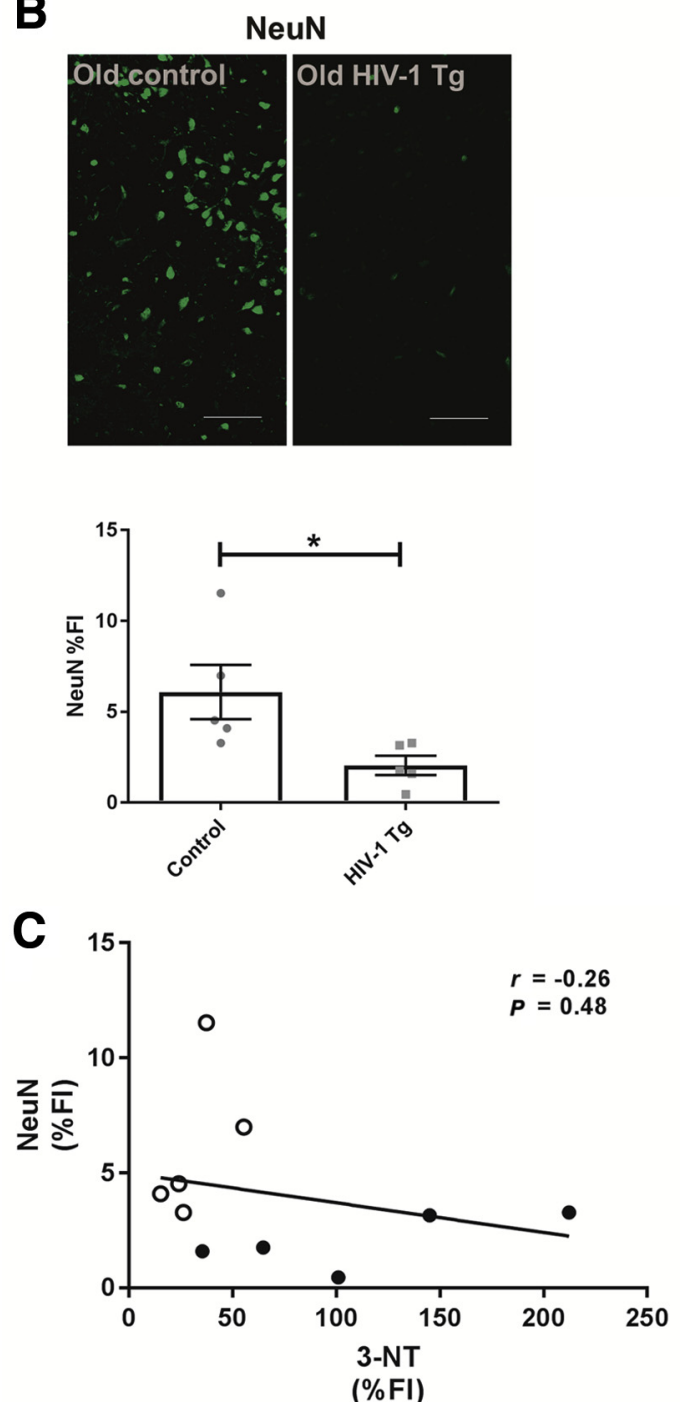

Interestingly, the increase in markers of nitrosative stress in these animals occurred with no corresponding increase in Nrf2 expression or the Nrf2-regulated Gsh and Trx antioxidant enzyme systems (Figure 1, B and D, and Table 1). Nrf2 is the major transcription factor regulating the biosynthesis, use, and regeneration of glutathione, TRX, and NADPH, thereby controlling the cellular redox activity. ${ }^{22} \mathrm{~A}$ previous report in the Tg rat describes a reduction, rather than an increase, in Nrf2 and heme oxygenase-1 expression in the whole-brain, cerebellum, and hippocampus lysates. ${ }^{49}$ No induction in Nrf2 expression or its dependent phase 2 antioxidants was found within the striatum. Thus, it is possible that the nigrostriatal dopaminergic dysfunction in aging HIV-1 rats is also a consequence of dysregulation in Nrf2 and redox adaptation, eventually failing to counteract the nitrosative stress affected by NOX4 and nNOS expression. This could, in turn, lead to the nitration of several targets, such as heat shock protein $90,{ }^{57}$ and the striatal neurofilament proteins, as we demonstrated, albeit only in a small sample size. An additional direct effect of Nrf2 expression imbalance could be neuronal cytotoxicity through the inhibition of cellular respiration and dysregulation of mitochondrial electron transport chain. ${ }^{21-24}$

These results support a model in which gp120 and/or Tat induce NOX4 expression and nNOS activation, which subsequently leads to ROS/RNS generation and nitrosative stress, protein tyrosine nitration, and neuronal injury; this neuronal pathology is influenced by aging and transgene status. The lack of effective antioxidant activity could further exacerbate the negative effects of OS/NS. This could eventually lead to the dopaminergic dysfunction that we and other groups have previously described. ${ }^{7,54,56,61-63,67,80-82}$ However, questions concerning the mechanism(s) by which Nrf2 activation is suppressed still need to be addressed, and our future work will concentrate on better understanding how Nrf2 is regulated in the HIV-1 Tg rat.

Ultimately, free radical damage to the brain is a process that is found to be common to the pathogenesis of HIVassociated neurologic disorders and many neurodegenerative diseases. Therefore, the identification of increased 3-NT modification of neurofilaments in dopaminergic neurons may be both a biological indicator and/or a contributor to the dopaminergic dysfunction in the HIV-1 $\mathrm{Tg}$ rat. We, therefore, propose the HIV $-1 \mathrm{Tg}$ rat as an appropriate model of OS/NS in the setting of exposure to HIV viral proteins.

Figure 5 Neuronal loss in the substantia nigra. A and B: Immunostaining in the substantia nigra of the old animal cohort for 3-nitrotyrosine (3-NT; $P=0.03 ; \mathbf{A})$ and $\operatorname{NeuN}(P=0.03 ; \mathbf{B})$. Quantification data are represented as percentage fluorescence intensity (\%FI) and analyzed by $t$-test. The $P$ values for control versus HIV-1 transgenic $(\mathrm{Tg})$ animals are indicated. C: Correlation of 3-NT (\%FI) with NeuN (\%FI) is shown. Pearson correlation coefficient $(r)$ and the $P$ values are indicated in the graph. 0 pen circles indicate control rats; closed circles, HIV-1 Tg rats. ${ }^{*} P<0.05$. Scale bars $=100 \mu \mathrm{m}(\mathbf{A}$ and $\mathbf{B})$. 


\section{Acknowledgments}

We thank Vincent Schram, Ph.D., and Lynne Holzclaw, B.S. (Microscopy and Imaging Core, Eunice Kennedy Shriver National Institute of Child Health and Human Development, NIH), for help and assistance; and Paul Wakim, Ph.D., for help in running the statistical analyses.

W.R. and D.A.H. are the guarantors of this work and, as such, had full access to all of the data in the study and take responsibility for the integrity of the data and the accuracy of the data analysis.

\section{References}

1. Farhadian S, Patel P, Spudich S: Neurological complications of HIV infection. Curr Infect Dis Rep 2017, 19:50

2. Eggers C, Arendt G, Hahn K, Husstedt IW, Maschke M, Neuen-Jacob E, Obermann M, Rosenkranz T, Schielke E, Straube E: HIV-1-associated neurocognitive disorder: epidemiology, pathogenesis, diagnosis, and treatment. J Neurol 2017, 264:1715-1727

3. McArthur JC, Steiner J, Sacktor N, Nath A: Human immunodeficiency virus-associated neurocognitive disorders: mind the gap. Ann Neurol 2010, 67:699-714

4. McArthur JC, Haughey N, Gartner S, Conant K, Pardo C, Nath A, Sacktor N: Human immunodeficiency virus-associated dementia: an evolving disease. J Neurovirol 2003, 9:205-221

5. Desplats P, Dumaop W, Smith D, Adame A, Everall I, Letendre S, Ellis R, Cherner M, Grant I, Masliah E: Molecular and pathologic insights from latent HIV-1 infection in the human brain. Neurology 2013, 80:1415-1423

6. Agrawal L, Louboutin JP, Reyes BA, Van Bockstaele EJ, Strayer DS: HIV-1 Tat neurotoxicity: a model of acute and chronic exposure, and neuroprotection by gene delivery of antioxidant enzymes. Neurobiol Dis 2012, 45:657-670

7. Hu S, Sheng WS, Lokensgard JR, Peterson PK, Rock RB: Preferential sensitivity of human dopaminergic neurons to gp120-induced oxidative damage. J Neurovirol 2009, 15:401-410

8. Mocchetti I, Bachis A, Avdoshina V: Neurotoxicity of human immunodeficiency virus-1: viral proteins and axonal transport. Neurotox Res 2012, 21:79-89

9. Bezzi P, Domercq M, Brambilla L, Galli R, Schols D, De Clercq E, Vescovi A, Bagetta G, Kollias G, Meldolesi J: CXCR4-activated astrocyte glutamate release via $\mathrm{TNF} \alpha$ : amplification by microglia triggers neurotoxicity. Nat Neurosci 2001, 4:702-710

10. Radja F, Kay DG, Albrecht S, Jolicoeur P: Oligodendrocyte-specific expression of human immunodeficiency virus type 1 Nef in transgenic mice leads to vacuolar myelopathy and alters oligodendrocyte phenotype in vitro. J Virol 2003, 77:11745-11753

11. Legrand-Poels S, Vaira D, Pincemail J, Van de Vorst A, Piette J: Activation of human immunodeficiency virus type 1 by oxidative stress. AIDS Res Hum Retroviruses 1990, 6:1389-1397

12. Boven LA, Gomes L, Hery C, Gray F, Verhoef J, Portegies P, Tardieu M, Nottet HS: Increased peroxynitrite activity in AIDS dementia complex: implications for the neuropathogenesis of HIV-1 infection. J Immunol 1999, 162:4319-4327

13. Nakamura H, Masutani H, Yodoi J: Redox imbalance and its control in HIV infection. Antioxid Redox Signal 2002, 4:455-464

14. Colado Simão AN, Victorino VJ, Morimoto HK, Reiche EM, Panis C: Redox-driven events in the human immunodeficiency virus type 1 (HIV-1) infection and their clinical implications. Curr HIV Res 2015, 13:143-150

15. Uzasci L, Nath A, Cotter R: Oxidative stress and the HIV-infected brain proteome. J Neuroimmune Pharmacol 2013, 8:1167-1180
16. Pang X, Panee J: Roles of glutathione in antioxidant defense, inflammation, and neuron differentiation in the thalamus of HIV-1 transgenic rats. J Neuroimmune Pharmacol 2014, 9:413-423

17. Steiner J, Haughey N, Li W, Venkatesan A, Anderson C, Reid R, Malpica T, Pocernich C, Butterfield DA, Nath A: Oxidative stress and therapeutic approaches in HIV dementia. Antioxid Redox Signal 2006, 8:2089-2100

18. Goodkin K, Miller EN, Cox C, Reynolds S, Becker JT, Martin E, Selnes OA, Ostrow DG, Sacktor NC: Effect of ageing on neurocognitive function by stage of HIV infection: evidence from the Multicenter AIDS Cohort Study. Lancet HIV 2017, 4:e411-e422

19. Goodkin K, Wilkie F, Concha M, Hinkin C, Symes S, Baldewicz T, Asthana D, Fujimura R, Lee D, Van Zuilen M: Aging and neuroAIDS conditions and the changing spectrum of HIV-1-associated morbidity and mortality. J Clin Epidemiol 2001, 54:S35-S43

20. Fields J, Dumaop W, Langford T, Rockenstein E, Masliah E: Role of neurotrophic factor alterations in the neurodegenerative process in HIV associated neurocognitive disorders. J Neuroimmune Pharmacol 2014, 9:102-116

21. Torreilles F, Salman-Tabcheh S, Guérin M-C, Torreilles J: Neurodegenerative disorders: the role of peroxynitrite. Brain Res Rev 1999, 30:153-163

22. Dinkova-Kostova AT, Abramov AY: The emerging role of Nrf2 in mitochondrial function. Free Radic Biol Med 2015, 88:179-188

23. Cassina A, Radi R: Differential inhibitory action of nitric oxide and peroxynitrite on mitochondrial electron transport. Arch Biochem Biophys 1996, 328:309-316

24. Brand MD: Mitochondrial generation of superoxide and hydrogen peroxide as the source of mitochondrial redox signaling. Free Radic Biol Med 2016, 100:14-31

25. Holmström KM, Finkel T: Cellular mechanisms and physiological consequences of redox-dependent signalling. Nat Rev Mol Cell Biol 2014, 15:411-421

26. Kishida KT, Klann E: Sources and targets of reactive oxygen species in synaptic plasticity and memory. Antioxid Redox Signal 2007, 9: $233-244$

27. Grivennikova VG, Vinogradov AD: Partitioning of superoxide and hydrogen peroxide production by mitochondrial respiratory complex I. Biochim Biophys Acta 2013, 1827:446-454

28. Cobb CA, Cole MP: Oxidative and nitrative stress in neurodegeneration. Neurobiol Dis 2015, 84:4-21

29. Block K, Gorin Y: Aiding and abetting roles of NOX oxidases in cellular transformation. Nat Rev Cancer 2012, 12:627-637

30. Ma MW, Wang J, Zhang Q, Wang R, Dhandapani KM, Vadlamudi RK, Brann DW: NADPH oxidase in brain injury and neurodegenerative disorders. Mol Neurodegener 2017, 12:7

31. Suzuki YJ, Forman HJ, Sevanian A: Oxidants as stimulators of signal transduction. Free Radic Biol Med 1997, 22:269-285

32. Nayernia Z, Jaquet V, Krause K-H: New insights on NOX enzymes in the central nervous system. Antioxid Redox Signal 2014, 20: 2815-2837

33. Pérez-Matute P, Pérez-Martínez L, Blanco J, Oteo J: Role of mitochondria in HIV infection and associated metabolic disorders: focus on nonalcoholic fatty liver disease and lipodystrophy syndrome. Oxid Med Cell Longev 2013, 2013:493413

34. Ischiropoulos $\mathrm{H}$ : Biological tyrosine nitration: a pathophysiological function of nitric oxide and reactive oxygen species. Arch Biochem Biophys 1998, 356:1-11

35. Sokolovsky M, Riordan JF, Vallee BL, Tetranitromethane: a reagent for the nitration of tyrosyl residues in proteins. Biochemistry 1966, 5: $3582-3589$

36. Radi R: Protein tyrosine nitration: biochemical mechanisms and structural basis of functional effects. Acc Chem Res 2013, 46: $550-559$

37. Sharma NM, Patel KP: Post-translational regulation of neuronal nitric oxide synthase: implications for sympathoexcitatory states. Expert Opin Ther Targets 2017, 21:11-22 
38. Weissman BA, Jones CL, Liu Q, Gross SS: Activation and inactivation of neuronal nitric oxide synthase: characterization of $\mathrm{Ca} 2+-$ dependent [125 I] calmodulin binding. Eur J Pharmacol 2002, 435: $9-18$

39. Zhang M, An C, Gao Y, Leak RK, Chen J, Zhang F: Emerging roles of Nrf2 and phase II antioxidant enzymes in neuroprotection. Prog Neurobiol 2013, 100:30-47

40. Cooke MS, Evans MD, Dizdaroglu M, Lunec J: Oxidative DNA damage: mechanisms, mutation, and disease. FASEB J 2003, 17: 1195-1214

41. Stark G: Functional consequences of oxidative membrane damage. J Membr Biol 2005, 205:1-16

42. Kensler TW, Wakabayashi N, Biswal S: Cell survival responses to environmental stresses via the Keap1-Nrf2-ARE pathway. Annu Rev Pharmacol Toxicol 2007, 47:89-116

43. Escartin C, Won SJ, Malgorn C, Auregan G, Berman AE, Chen P-C, Déglon N, Johnson JA, Suh SW, Swanson RA: Nuclear factor erythroid 2-related factor 2 facilitates neuronal glutathione synthesis by upregulating neuronal excitatory amino acid transporter 3 expression. J Neurosci 2011, 31:7392-7401

44. Aoyama K, Watabe M, Nakaki T: Regulation of neuronal glutathione synthesis. J Pharmacol Sci 2008, 108:227-238

45. Jornstedt MB, Kumar S, Holmgren A: [22] Selenite and selenodiglutathione: reactions with thioredoxin systems. Methods Enzymol 1995, 252:209-219

46. Zhong L, Arnér ES, Holmgren A: Structure and mechanism of mammalian thioredoxin reductase: the active site is a redox-active selenolthiol/selenenylsulfide formed from the conserved cysteineselenocysteine sequence. Proc Natl Acad Sci U S A 2000, 97: $5854-5859$

47. Silva-Adaya D, Gonsebatt ME, Guevara J: Thioredoxin system regulation in the central nervous system: experimental models and clinical evidence. Oxid Med Cell Longev 2014, 2014:590808

48. Burke-Gaffney A, Callister ME, Nakamura H: Thioredoxin: friend or foe in human disease? Trends Pharmacol Sci 2005, 26:398-404

49. Davinelli S, Scapagnini G, Denaro F, Calabrese V, Benedetti F, Krishnan S, Curreli S, Bryant J, Zella D: Altered expression pattern of Nrf2/HO-1 axis during accelerated-senescence in HIV-1 transgenic rat. Biogerontology 2014, 15:449-461

50. Vigorito M, Cao J, Li MD, Chang SL: Acquisition and long-term retention of spatial learning in the human immunodeficiency virus-1 transgenic rat: effects of repeated nicotine treatment. J Neurovirol 2013, 19:157-165

51. Moran LM, Booze RM, Mactutus CF: Time and time again: temporal processing demands implicate perceptual and gating deficits in the HIV-1 transgenic rat. J Neuroimmune Pharmacol 2013, 8:988-997

52. Peng J, Vigorito M, Liu X, Zhou D, Wu X, Chang S: The HIV-1 transgenic rat as a model for HIV-1 infected individuals on HAART. J Neuroimmunol 2010, 218:94-101

53. Reid W, Sadowska M, Denaro F, Rao S, Foulke J, Hayes N, Jones O, Doodnauth D, Davis H, Sill A: An HIV-1 transgenic rat that develops HIV-related pathology and immunologic dysfunction. Proc Natl Acad Sci U S A 2001, 98:9271-9276

54. Reid WC, Casas R, Papadakis GZ, Muthusamy S, Lee DE, Ibrahim WG, Nair A, Koziol D, Maric D, Hammoud DA: Neurobehavioral abnormalities in the HIV-1 transgenic rat do not correspond to neuronal hypometabolism on 18F-FDG-PET. PLoS One 2016, 11:e0152265

55. Lashomb AL, Vigorito M, Chang SL: Further characterization of the spatial learning deficit in the human immunodeficiency virus-1 transgenic rat. J Neurovirol 2009, 15:14-24

56. Moran L, Booze R, Webb K, Mactutus C: Neurobehavioral alterations in HIV-1 transgenic rats: evidence for dopaminergic dysfunction. Exp Neurol 2013, 239:139-147

57. Cho Y-E, Lee M-H, Song B-J: Neuronal cell death and degeneration through increased nitroxidative stress and tau phosphorylation in HIV-1 transgenic rats. PLoS One 2017, 12:e0169945
58. Song G, Nesil T, Cao J, Yang Z, Chang SL, Li MD: Nicotine mediates expression of genes related to antioxidant capacity and oxidative stress response in HIV-1 transgenic rat brain. J Neurovirol 2016, 22:114-124

59. Pang X, Panee J, Liu X, Berry MJ, Chang SL, Chang L: Regional variations of antioxidant capacity and oxidative stress responses in HIV-1 transgenic rats with and without methamphetamine administration. J Neuroimmune Pharmacol 2013, 8:691-704

60. Villeneuve LM, Purnell PR, Stauch KL, Callen SE, Buch SJ, Fox HS: HIV-1 transgenic rats display mitochondrial abnormalities consistent with abnormal energy generation and distribution. J Neurovirol 2016 , 22:564-574

61. Lee DE, Reid WC, Ibrahim WG, Peterson KL, Lentz MR, Maric D, Choyke PL, Jagoda EM, Hammoud DA: Imaging dopaminergic dysfunction as a surrogate marker of neuropathology in a smallanimal model of HIV. Mol Imaging 2014, 13:1-10

62. Reid WC, Ibrahim WG, Kim SJ, Denaro F, Casas R, Lee DE, Maric D, Hammoud DA: Characterization of neuropathology in the HIV-1 transgenic rat at different ages. J Neuroimmunol 2016, 292: $116-125$

63. Sinharay S, Lee D, Shah S, Muthusamy S, Papadakis GZ, Zhang X, Maric D, Reid WC, Hammoud DA: Cross-sectional and longitudinal small animal PET shows pre and post-synaptic striatal dopaminergic deficits in an animal model of HIV. Nucl Med Biol 2017, 55:27-33

64. Shah S, Cannon JR, Fenselau C, Briken V: A duplicated ESAT-6 region of ESX-5 is involved in protein export and virulence of mycobacteria. Infect Immun 2015, 83:4349-4361

65. Paxinos G, Watson C: The Rat Brain in Stereotaxic Coordinates. ed 6. London, UK, Academic Press, Elsevier Inc., 2007

66. Zhang Y, Wang M, Li H, Zhang H, Shi Y, Wei F, Liu D, Liu K, Chen D: Accumulation of nuclear and mitochondrial DNA damage in the frontal cortex cells of patients with HIV-associated neurocognitive disorders. Brain Res 2012, 1458:1-11

67. Agrawal L, Louboutin JP, Marusich E, Reyes BA, Van Bockstaele EJ, Strayer DS: Dopaminergic neurotoxicity of HIV-1 gp120: reactive oxygen species as signaling intermediates. Brain Res 2010, 1306:116-130

68. Banerjee A, Zhang X, Manda KR, Banks WA, Ercal N: HIV proteins (gp120 and Tat) and methamphetamine in oxidative stress-induced damage in the brain: potential role of the thiol antioxidant N-acetylcysteine amide. Free Radic Biol Med 2010, 48:1388-1398

69. Corasaniti MT, Strongoli MC, Piccirilli S, Nistico R, Costa A, Bilotta A, Turano P, Finazzi-Agro A, Bagetta G: Apoptosis induced by gp120 in the neocortex of rat involves enhanced expression of cyclooxygenase type 2 and is prevented by NMDA receptor antagonists and by the 21-aminosteroid U-74389G. Biochem Biophys Res Commun 2000, 274:664-669

70. Louboutin JP, Agrawal L, Reyes BA, Van Bockstaele EJ, Strayer DS: HIV-1 gp120-induced injury to the blood-brain barrier: role of metalloproteinases 2 and 9 and relationship to oxidative stress. J Neuropathol Exp Neurol 2010, 69:801-816

71. Louboutin JP, Strayer DS: Blood-brain barrier abnormalities caused by HIV-1 gp120: mechanistic and therapeutic implications. ScientificWorldJournal 2012, 2012:482575

72. Price TO, Ercal N, Nakaoke R, Banks WA: HIV-1 viral proteins gp120 and Tat induce oxidative stress in brain endothelial cells. Brain Res 2005, 1045:57-63

73. Price TO, Uras F, Banks WA, Ercal N: A novel antioxidant N-acetylcysteine amide prevents gp120- and Tat-induced oxidative stress in brain endothelial cells. Exp Neurol 2006, 201:193-202

74. Ronaldson PT, Bendayan R: HIV-1 viral envelope glycoprotein gp120 produces oxidative stress and regulates the functional expression of multidrug resistance protein-1 (Mrp1) in glial cells. J Neurochem 2008, 106:1298-1313

75. Aksenov MY, Hasselrot U, Wu G, Nath A, Anderson C, Mactutus CF, Booze RM: Temporal relationships between HIV-1 Tat-induced neuronal degeneration, OX-42 immunoreactivity, 
reactive astrocytosis, and protein oxidation in the rat striatum. Brain Res 2003, 987:1-9

76. Flora G, Lee YW, Nath A, Hennig B, Maragos W, Toborek M: Methamphetamine potentiates HIV-1 Tat protein-mediated activation of redox-sensitive pathways in discrete regions of the brain. Exp Neurol 2003, 179:60-70

77. New DR, Maggirwar SB, Epstein LG, Dewhurst S, Gelbard HA: HIV-1 Tat induces neuronal death via tumor necrosis factor-alpha and activation of non-N-methyl-D-aspartate receptors by a NFkappaBindependent mechanism. J Biol Chem 1998, 273:17852-17858

78. Pocernich CB, Sultana R, Mohmmad-Abdul H, Nath A, Butterfield DA: HIV-dementia, Tat-induced oxidative stress, and antioxidant therapeutic considerations. Brain Res Brain Res Rev 2005, 50:14-26

79. Toborek M, Lee YW, Pu H, Malecki A, Flora G, Garrido R, Hennig B, Bauer HC, Nath A: HIV-Tat protein induces oxidative and inflammatory pathways in brain endothelium. J Neurochem 2003, 84:169-179

80. Wallace DR, Dodson S, Nath A, Booze RM: Estrogen attenuates gp120- and tat1-72-induced oxidative stress and prevents loss of dopamine transporter function. Synapse 2006, 59:51-60

81. Lentz MR, Peterson KL, Ibrahim WG, Lee DE, Sarlls J, Lizak MJ, Maric D, Reid WC, Hammoud DA: Diffusion tensor and volumetric magnetic resonance measures as biomarkers of brain damage in a small animal model of HIV. PLoS One 2014, 9:e105752

82. Casas R, Muthusamy S, Wakim PG, Sinharay S, Lentz MR, Reid WC, Hammoud DA: MR brain volumetric measurements are predictive of neurobehavioral impairment in the HIV-1 transgenic rat. Neuroimage Clin 2018, 17:659-666

83. Ivanov AV, Valuev-Elliston VT, Ivanova ON, Kochetkov SN, Starodubova ES, Bartosch B, Isaguliants MG: Oxidative stress during HIV infection: mechanisms and consequences. Oxid Med Cell Longev 2016, 2016:8910396
84. Kozieł R, Pircher H, Kratochwil M, Lener B, Hermann M, Dencher NA, Jansen-Dürr P: Mitochondrial respiratory chain complex I is inactivated by NADPH oxidase Nox4. Biochem J 2013, 452: 231-239

85. Boveris A, Valdez LB, Zaobornyj T, Bustamante J: Mitochondrial metabolic states regulate nitric oxide and hydrogen peroxide diffusion to the cytosol. Biochim Biophys Acta 2006, 1757:535-542

86. Wallace DC: A mitochondrial paradigm of metabolic and degenerative diseases, aging, and cancer: a dawn for evolutionary medicine. Annu Rev Genet 2005, 39:359-407

87. Lustgarten MS, Bhattacharya A, Muller FL, Jang YC, Shimizu T, Shirasawa T, Richardson A, Van Remmen H: Complex I generated, mitochondrial matrix-directed superoxide is released from the mitochondria through voltage dependent anion channels. Biochem Biophys Res Commun 2012, 422:515-521

88. Dunn JD, Alvarez LA, Zhang X, Soldati T: Reactive oxygen species and mitochondria: a nexus of cellular homeostasis. Redox Biol 2015, 6:472-485

89. Han D, Antunes F, Canali R, Rettori D, Cadenas E: Voltagedependent anion channels control the release of the superoxide anion from mitochondria to cytosol. J Biol Chem 2003, 278:5557-5563

90. Bonavia R, Bajetto A, Barbero S, Albini A, Noonan DM, Schettini G: HIV-1 Tat causes apoptotic death and calcium homeostasis alterations in rat neurons. Biochem Biophys Res Commun 2001, 288:301-308

91. Kone B: Protein-protein interactions controlling nitric oxide synthases. Acta Physiol Scand 2000, 168:27-31

92. Holden C, Haughey N, Nath A, Geiger J: Role of Na+/H+ exchangers, excitatory amino acid receptors and voltage-operated $\mathrm{Ca}$ $2+$ channels in human immunodeficiency virus type 1 gp120mediated increases in intracellular $\mathrm{Ca} 2+$ in human neurons and astrocytes. Neuroscience 1999, 91:1369-1378 